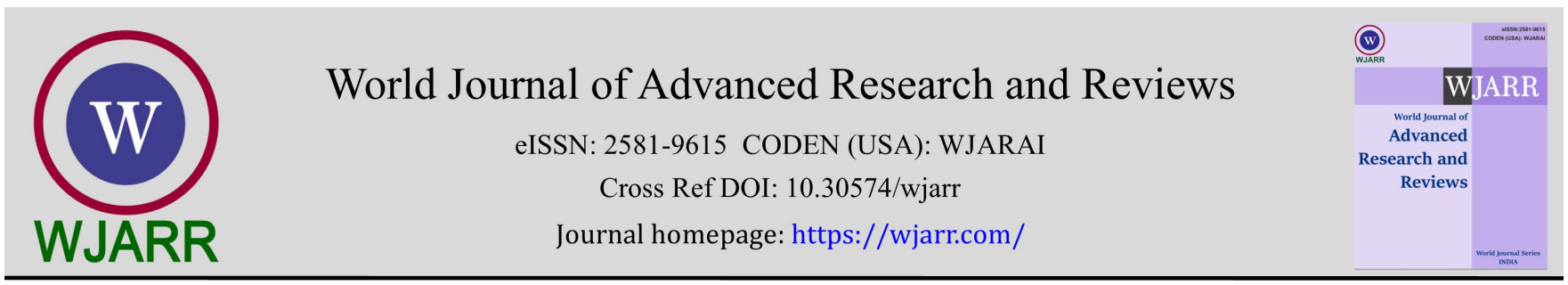

(REVIEW ARTICLE)

\title{
Behavioral dynamics of pedestrian's movements: Theoretical aspects of commercial arterial road frontage activities
}

\author{
Jacob Oluwoye * \\ College of Agricultural, Life and Natural Sciences, Department of Community and Regional Planning, Alabama A\&M \\ University, Huntsville, Alabama, USA.
}

World Journal of Advanced Research and Reviews, 2021, 11(03), 201-205

Publication history: Received on 04 August 2021; revised on 14 September 2021; accepted on 16 September 2021

Article DOI: https://doi.org/10.30574/wjarr.2021.11.3.0429

\begin{abstract}
The common scientific approaches to the reasoning of problems are mathematical reasoning or statistical reasoning. Mathematical or formal reasoning is usually deductive, therein one reason from general assumptions to specifics using symbolic logic and axioms for multi criteria decision-making. Mathematical probability, which is the basis of all statistical theories, had its beginning in the past. The aim of this paper is to explore a number of the mathematical and statistical aspects of the disposition and behavior of road frontage activities, which are of importance in pedestrian behavior as considered. It's shown that number of crossings from right to left is proportional to the pedestrian on the right $(\mathrm{PXRL} \propto \mathrm{NR})$ and therefore, the number of crossings left to right is proportional to the pedestrians on the left $(P X L R \propto N L)$. Frequency distributions of the pedestrians generated for a given shopping string arterial were of 4 kinds, one related to pedestrians passing through not crossing the road, not going into and out of outlets. The second kind related to pedestrians crossing the road for the aim of going into and out of outlets, etc. the third kind related to pedestrian going into shops and eventually, the fourth kind related to others, e.g. Pedestrians generated from parking vehicles, buses, etc. A formula is given for the frequency with crossing from left to right and right to leave based on the land-use activities on the left and right. In considering the capacity of road systems it should be remembered that increases in traffic flow generally produce corresponding decreases in speed. However, it's an assumption that a rise in population generated along the footpath can cause the crossing the road, and usually produce corresponding decreases in vehicle speed. The paper concludes with a constatation of the pedestrian movements at a continuing rate that expressed in mathematical form.
\end{abstract}

Keywords: Roads; Traffic; Theoretical; Pedestrian movements; Behavior; Road frontage

\section{Introduction}

The common scientific approaches to the reasoning of problems are mathematical reasoning or statistical reasoning. Mathematical or formal reasoning is usually deductive, therein one reason from general assumptions to specifics using symbolic logic and axioms for multi-criteria decision-making. Mathematical probability, which is that the basis of all statistical theory, had its beginning in past. The aim of this paper is to explore a number of the mathematical and statistical aspects of the disposition and behavior of road frontage activities, which are of importance in pedestrian behavior as considered. it's shown that number of crossings from right to left is proportional to the pedestrian on the right (PXRL $\propto N R$ ) and therefore the number of crossings left to right is proportional to the pedestrians on the left (PXLR $\propto \mathrm{NL})$.

\footnotetext{
* Corresponding author: Jacob Oluwoye

College of Agricultural, Life and Natural Sciences, Department of Community and Regional Planning, Alabama A\&M University, Huntsville, Alabama, USA.
}

Copyright (C) 2021 Author(s) retain the copyright of this article. This article is published under the terms of the Creative Commons Attribution Liscense 4.0. 
Since the rapid development of urbanization and industrialization, the increasing use of automobiles, bicycles, on urban road systems, also as other relevant problems, have made the road systems most of the developed and underdeveloped countries more crowded [1]. Within the Third World and fewer developed countries there are sections of the road, subjected to heavy traffic flow, aligned by poor building stock, containing mixed land-use, barren of landscaping, with discordant power poles and street structures. [2] reported that the broad nature of the road and therefore the road environment within the Third World countries are seen as: * Points of traffic jam where the road has insufficient capacity to deal with existing traffic volumes. * Pedestrian movements -- there's no provision for zebra crossings, no island or refuge, no sidewalk along the road. * Unpleasant living, shopping, and dealing environments thanks to noise and pollution. * Inadequate provision for off-street parking and loading facilities, largely caused by historical circumstances and lack of foresight and investment within the facilities. * Use of public streets for casual activities related to adjoining properties. * Placement of informal activities on the carriageway. * Parking of cars along the road obstructing pedestrians and resulting in a visually unpleasant environment, danger, and lack of visibility. * Reduced amenity of adjoining residential development in side streets thanks to on street parking and traffic movements. ${ }^{*}$ Traffic conflicts/accidents and hazards created by having retail/commercial/industrial development ashore fronting commercial roads and requiring vehicle access thereto. Furthermore, most roads have movement and access functions. Conflicts arise where roads with heavy traffic penetrate areas intended to be precincts; this is often described within the paper because the problem of roads in environments. Conflicts also arise where traffic routes have active frontage activities: this is often seen because the problem of roads as environments. Notwithstanding, some researchers explained that when the footpath becomes crowded, people are more likely to steer within the carriageway [3][4][5]. Risk decreases with increase in density [6]; [7]. Another researcher explained pedestrian interference with traffic when leaving a cinema [8].

\section{Conceptual Theory of Arterial Road Frontage Activities}

The theory developed in this paper as follows:

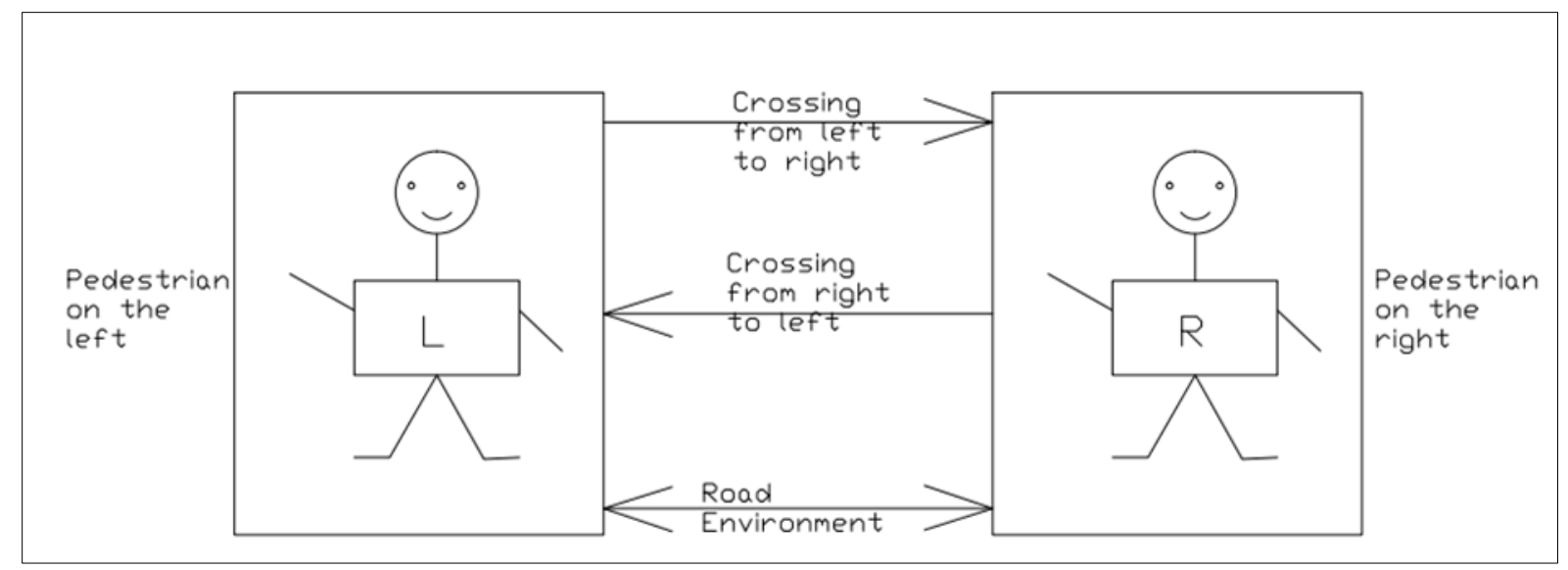

Figure 1a A mode of containing three objects, and two binary relations on road space (Ped from left to right) 


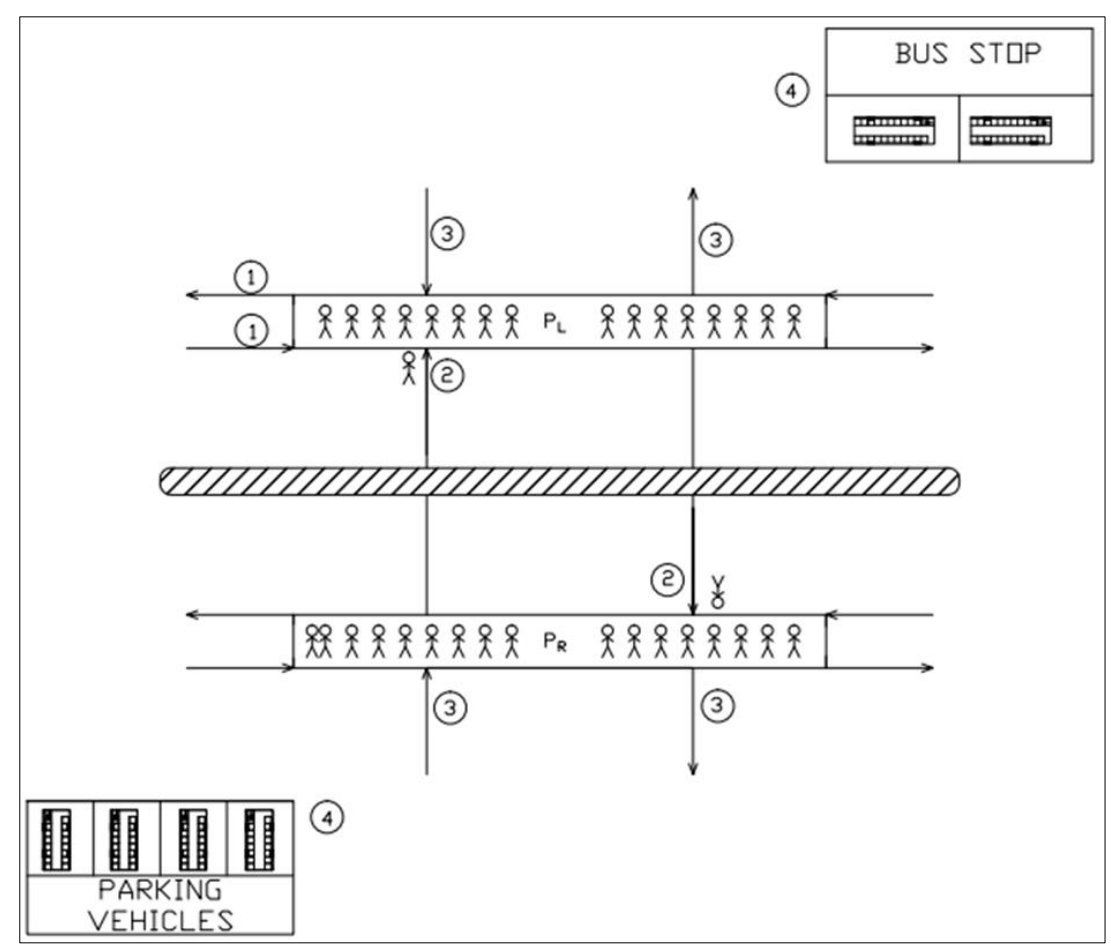

Figure 1b Conceptual Framework of Birth and Death of Roads and Road Environment Research

\section{Analysis of Framework}

HL Westerman [5] reported that most roads have movement and access functions. Conflicts arise where roads with heavy traffic penetrate into areas intended to be precincts; this is described in the paper as the problem of roads in environments. Conflicts also arise where traffic routes have active frontage development: this is seen as the problem of roads as environments.

For each of the PL and PR there are several inputs and outputs. e.g.

- EW, WE: Pedestrian passing through, not crossing road and not going into and out of shops

- $\quad$ NS, SN: Pedestrian crossing road

- NS, SN: Pedestrians going into and out of shops etc.

- EW, WE pedestrian going into shops

- Others: Pedestrians generated from parking vehicles, buses etc.

By looking at set-down and pick up (\#4 and \#5), probably time-dependent ie. Peak/non-peak

By looking at in/out for shops, etc office-like Bank, Educational offices and probably time-dependent - again peak/nonpeak shops probably have a very small net influence on (\#3).

The flows in the EW and WE direction may depend on things like location of bus stops and market area during market day. These may be a small tidal effect on (\#1) crossing and through pedestrian.

Assumption - A basic assumption of the above problem is that pedestrian movements at constant rate and can be expressed in mathematical form.

$$
\begin{aligned}
& P L(t+\delta t)=P L(t)+P X R L(\delta t)-P X L R(\delta t) \\
& N_{L}(t+\delta t)=N_{L}(t)+\lambda_{R L} \delta t N_{R}(t)-\lambda_{L R} \delta t_{N}(t) \\
& N_{R}(t+\delta t)=N_{R}(t)+\lambda_{L R} \delta t N_{L}(t)-\lambda_{R L} \delta t N_{R}(t)
\end{aligned}
$$


$\mathrm{N}^{1} \mathrm{~L}(\mathrm{t})=\lambda_{\mathrm{RL}} \mathrm{N}_{\mathrm{R}}(\mathrm{t})-\lambda_{\mathrm{LR}} \mathrm{N}_{\mathrm{L}}(\mathrm{t})$

$\mathrm{N}^{1}{ }_{\mathrm{R}}(\mathrm{t})=\lambda_{\mathrm{LR}} \mathrm{N}_{\mathrm{L}}(\mathrm{t})-\lambda_{\mathrm{RL}} \mathrm{N}_{\mathrm{R}}(\mathrm{t})$

Adding complies $\mathrm{N}_{\mathrm{L}}(\mathrm{t})+\mathrm{N}_{\mathrm{R}}(\mathrm{t})=\mathrm{N}$

$N^{\prime \prime}{ }_{L}(t)=\lambda_{R L} N_{R}^{\prime}(t)-\lambda_{L R} N^{\prime} L(t)=\lambda_{R L}$

$N^{\prime}{ }_{L}(t)=\lambda_{R L}\left(N-N_{L}(t)\right)-\lambda_{L R} N_{L}(t)$

$=N \lambda_{R L}-\left(\lambda_{R L}+\lambda_{L R}\right) N_{L}(t)$

$N^{\prime}{ }_{L}(t)+\lambda N_{L}(t)=0$

$A+B e^{-(\lambda R L+\lambda L R) t}=A+B e^{-\lambda t}$

$-\lambda \mathrm{Be}^{-\lambda t}=\mathrm{N} \lambda_{\mathrm{RL}}-\lambda(\mathrm{A}+\mathrm{Be}-\lambda \mathrm{t})$

$$
\mathrm{A}=\frac{\lambda_{R L}}{\lambda_{R L}+\lambda_{L R}}
$$

$N_{L}(t)=\mathrm{N} \frac{\lambda_{R L}}{\lambda_{R L}+\lambda_{L R}}+B_{e^{-\left(\lambda_{R L}+\lambda_{L R}\right) t}}$

$N_{L}(0)=\mathrm{N} \frac{\lambda_{R L}}{\lambda_{R L}+\lambda_{L R}}+B$

$\therefore \mathrm{B}=N_{L}(0)-\mathrm{N} \frac{\lambda_{R L}}{\lambda_{R L}+\lambda_{L R}}$

$$
N_{L}(t)=\mathrm{N} \frac{\lambda_{R L}}{\lambda_{R L}+\lambda_{L R}}+\left(N_{L}(0)-\mathrm{N} \frac{\lambda_{R L}}{\lambda_{R L}+\lambda_{L R}}\right)^{e^{-\left(\lambda_{R L}+\lambda_{L R}\right) t}}
$$

The point is $\mathrm{N}_{\mathrm{L}}(\mathrm{t})=\mathrm{A}+\mathrm{Be} \mathrm{e}^{-\lambda t}$ have assumed number of crossing right to left is proportional to pedestrian on the right.

$P_{X L R} \propto N_{R}$

Number of crossing left to right is proportional to pedestrian on the left

$P_{X L R} \propto N_{L}$

$\therefore$ Number crossing $\mathrm{LR}=\mathrm{C}\left(A+B_{e^{-\lambda t}}\right)$

$=\left(A^{I}+B^{I}{ }^{-\lambda t}\right)$

Number crossing $R L=\left(A^{I I}+B^{I I} e^{-\lambda t}\right)$

Number crossing total $=\left(\bar{A}+\bar{B}_{e^{-\lambda t}}\right)$

\section{Conclusion}

In conclusion, the above analysis applies to steady state only. However, the of conflict observations is to determine the critical situations in traffic. This means that one seeks to obtain an insight into the probability of an accident as well as 
the seriousness of it. The question is how great the risk is and which elements are the most relevant from the above conceptual framework. Thus, there is a need for minimum vehicular and pedestrian volume warrants for the installation of pedestrian movement and crossings among commercial Arteria roads.

\section{Compliance with ethical standards}

\section{Acknowledgments}

I acknowledge the support of the Professor HL. Westerman and Dr.MC. Dunne for exposing me to the conceptual models of multifunctional roads and road environments.

\section{References}

[1] Oluwoye J0. An investigation of the factors of the design of roads that affect planning for traffic (Pedestrian) safety in the third world. SATC. 2000.

[2] Oluwoye JO. Planning for Road Safety in Nigeria, Form/Work an Interdisciplinary Journal of Design and the Built Environment. No.1 October 1997; 22-33.

[3] SJ Older. Movement of Pedestrians on Footways in Shopping Streets, Traffic Engineering and Control. Aug. 1968; 160-163.

[4] JO Oluwoye. Assessment of pedestrian crossing activity in the determination of reducing conflict between pedestrians and vehicles along a trip commercial streets in Nigeria, PhD Thesis May. 1988.

[5] HL Westerman. Multi-functional roads and road environments in, Australia:Unpublished paper, School of Town Planning, UNSW, NSW. 1984.

[6] AM Mackie, SJ Older. Study of Pedestrian Risk in Crossing Busy Roads in London Inner Suburbs, Traffic Engineering and Control. May 1965; 376-380.

[7] J0 Oluwoye. Individual Group Perceptions of Risk in Crossing an Arterial Road in Developing Countries, The Second National Conference of the Risk Engineering Society. 2-3 Oct 1997.

[8] A Cedar. Pedestrian/Traffic Interactions: An Algorithm to Assign Pedestrian Groups Dispersing at Public Gatherings", Proceedings of International Conference on Pedestrian Safety 1976 Michel Haifa. 1-8, 20-23. 